\title{
Joint Loading in the Lower Extremities during Elliptical Exercise
}

\author{
TUNG-WU LU ${ }^{1}$, HUI-LIEN CHIEN ${ }^{1}$, and HAO-LING CHEN ${ }^{2}$ \\ ${ }^{1}$ Institute of Biomedical Engineering and ${ }^{2}$ School of Occupational Therapy, National Taiwan University, TAIWAN
}

\begin{abstract}
LU, T.W., H.L. CHIEN, and H.L. CHEN. Joint Loading in the Lower Extremities during Elliptical Exercise. Med. Sci. Sports Exerc., Vol. 39, No. 9, pp. 1651-1658, 2007. Purpose: To determine the joint loading during elliptical exercise (EE) by a detailed three-dimensional dynamic analysis, and to compare the results with those during level walking. Methods: Fifteen male adults performed level walking and EE while 3D kinematic data, right pedal reaction forces (PRF), and ground reaction forces (GRF) were measured. Pedal rate (cadence) and step length during EE without workload were set according to those measured during level walking for each subject. The motion of the body's center of mass, lower-limb-joint angles and moments were obtained. Results: Pedal rates and step lengths were $52.20 \mathrm{rpm}(\mathrm{SD}=2.34)$ and $50.56 \mathrm{~cm}(\mathrm{SD}=2.14)$, respectively. During early stance the vertical PRF was smaller than the GRF, and the medial and posterior shear components were greater. PRF also occurred during swing. Loading rates around heelstrike during EE were all smaller than those during walking. During EE, the peak flexion angles of the hip, knee and ankle were greater. Peak hip flexor and knee extensor moments were also greater, whereas peak ankle plantarflexor moments and all abductor moments were smaller. Conclusions: Different lower-limb kinematics and kinetics were found between EE and level walking. Smaller vertical PRF and loading rates during EE were achieved at the expense of greater hip flexor and knee extensor moments. Use of the elliptical trainer for athletic and rehabilitative training would have to consider users' joint function and muscle strength, especially at the knee, to avoid injuries. Key Words: ELLIPTICAL TRAINER, FITNESS, KINEMATICS, KINETICS, LOWER LIMBS
\end{abstract}

$\mathrm{E}$ xercise with an elliptical trainer (ET) has been shown to be beneficial for the development and maintenance of cardiorespiratory fitness by providing an adequate metabolic challenge $(11,16)$. Physiological improvements after training using ET were also found to be similar to those using a treadmill and stair-climber (11). These findings suggest that elliptical exercise (EE) can be developed as a low-impact aerobic exercise modality. In fact, EE training has increased in popularity over the last decade. According to an analysis by the Sporting Goods Manufacturers Association (SGMA) of the United States published in 2006, the population of ET users grew by $170 \%$ from 2000 to 2005 , an increase of nearly 10 million (23). Health clubs and individuals have purchased these machines to provide an option of fitness training that can be incorporated into an exercise regimen (3). Recently, EE has also been included for fitness training in the rehabilitation

\footnotetext{
Address for correspondence: Tung-Wu Lu, DPhil, Institute of Biomedical Engineering, National Taiwan University, No. 1, Sec. 1, Jen-Ai Road, Taipei 100, Taiwan, R.O.C.; E-mail: twlu@ntu.edu.tw.

Submitted for publication December 2006.

Accepted for publication May 2007.

0195-9131/07/3909-1651/0

MEDICINE \& SCIENCE IN SPORTS \& EXERCISE ${ }_{\circledast}$ Copyright $(C 2007$ by the American College of Sports Medicine

DOI: $10.1249 / \mathrm{mss} .0 \mathrm{~b} 013 \mathrm{e} 3180 \mathrm{dc} 9970$
}

programs of patients who have undergone hip arthroscopy (24), ACL revision surgery (25), or patellar tendonectomy (21), or who suffer from patellofemoral pain syndrome (12) or diabetes (10). Despite the benefit in cardiorespiratory fitness, the rapid growth in the number of ET and their wide clinical applications, little is known regarding the loading applied to the lower-limb joints during EE. Considering the lower impact nature of ET, the loading to the lower limbs may be different from walking. Green et al. (14) found that for similar intensity, the ratings of perceived exertion (RPE) of the legs during EE were significantly greater than those during treadmill exercise, although the overall RPE were similar. Increased loading at a joint and of the surrounding muscles or muscle groups may lead to early fatigue, which could in turn limit the duration of the exercise. Sufficient and appropriate duration of training is important for cardiorespiratory training (2) as exercise duration and intensity interact to achieve a fitness goal. On the other hand, the forces transmitted in the lower limbs are also closely related to potential musculoskeletal overuse injuries. Therefore, knowledge of the loading in the lower limbs during $\mathrm{EE}$ is essential to ensure an efficient and safe exercise environment, especially for patients.

ET provide walking-like exercise with reduced impact loading. Walking has been shown to have positive effects on health (22). However, the common daily activity of walking is not as smooth and harmless as has previously been believed (9). It has generally been accepted that the motion of the human body during walking is controlled in 


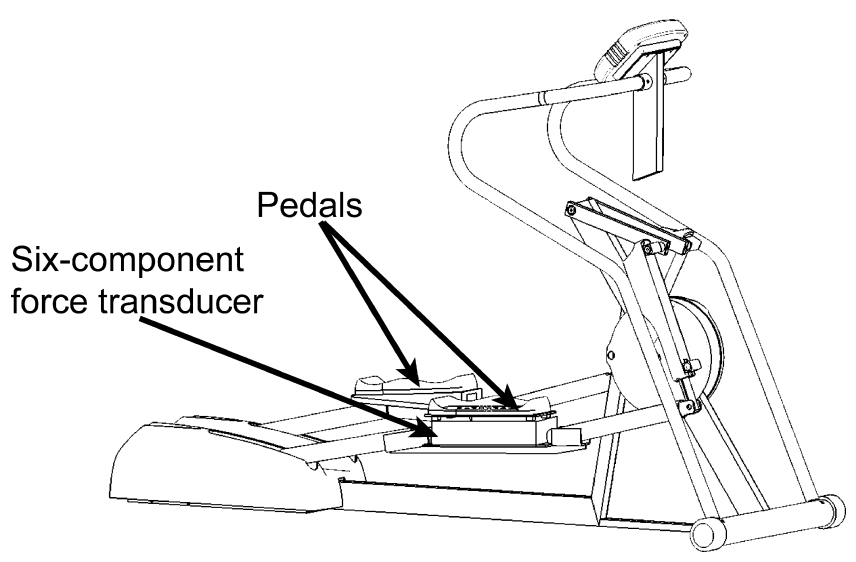

FIGURE 1-Experimental set-up showing the elliptical trainer and a six-component force transducer instrumented under the right pedal.

such a way so as to minimize the amount of mechanical energy required (4). With this goal, each joint of the leg is close to the line of action of the ground reaction force (GRF) to minimize the net external joint moments and thus reduce the muscle forces necessary to counteract these moments (7). The GRF that identifies the mechanical energy fluctuations displays an " $M$ "-shaped curve in the vertical component. While smooth during most of the stance phase, large repetitive impact forces occur at heelstrike. Repetitive impact loading is a likely source of deleterious stress (9). Therefore, a major disadvantage of level walking is that repetitive vertical impact forces at heelstrike may induce musculoskeletal injuries such as knee osteoarthritis (9). These impact forces also increase with increasing speed (28). EE improves these negative effects (impact forces) by maintaining both feet in constant contact with the pedal platforms to form a closed kinetic chain during the exercise. This reduces not only impact forces around heelstride at the stance limb, but also the magnitude of the GRF owing to the weight sharing from the swing leg. This is potentially an advantage over ground walking, during which the body is supported solely by the stance limb for about $66 \%$ of the stance phase. However, reduced GRF may not necessarily reduce the net external joint moments, as the posture of the lower limb may affect the lever arms available to the GRF at the joints. Detailed mechanical analysis is needed for a better understanding of the loading in the lower limbs during EE.

The motion of EE resembles that of standing cycling (14) in that the user applies force with alternate legs to pedals while the body is supported mainly by the lower limbs. Both exercises are closed kinetic chain exercises with each leg following elliptical-shaped trajectories of the pedals during EE and circular-shaped ones during standing cycling. Even with impact forces much lower than walking and running, cyclists suffer from a considerable number of injuries, the most common of which seem to be at the knee (6). This indicates that these injuries are not related to impact forces. Studies of cycling have shown that inappropriate pedal design and ankle positioning increase stresses at the knee $(5,6)$. During EE, the feet are constrained by pedals to follow an elliptical trajectory, with the possibility of producing disadvantageous joint loads.

Previous studies on EE have been limited and have focused mostly on its effects on the cardiorespiratory function $(3,11,14)$. To the best knowledge of the authors, no study has ever quantified the forces transmitted in the lower limbs during EE, and compared them with those during level walking for a better understanding of the benefits of $\mathrm{EE}$ and its potential risk of injuries to the musculoskeletal system. The purpose of the study was to determine the joint loading during EE through a detailed 3D dynamic analysis of the lower extremities and a comparison of the results with those during level walking. Complete knowledge of the biomechanics of lower-limb joints will be helpful for the improvement of the design and the establishment of guidelines for safe use of the ET in the training of healthy users, as well as of patients during the course of rehabilitation.

\section{MATERIALS AND METHODS}

Subjects. Fifteen male adult volunteers (age: $25.5 \pm$ $2.5 \mathrm{yr}$; height: $173.7 \pm 4.9 \mathrm{~cm}$; mass: $72.7 \pm 7.9 \mathrm{~kg}$ ) participated in the current study. They were free from any musculoskeletal, cardiovascular, or neurological pathology, or any other disorders that might have affected the activity. Each subject gave written informed consent following the guidelines of the institutional human research ethics committee.

Instruments. A commercially available ET (Cycling \& Health Tech Industry R\&D Center, Taiwan) was used in this study (Fig. 1). For the measurement of the pedal reaction force (PRF) and its point of application, the right pedal of the ET was instrumented with a six-component force transducer (Bertec Corporation) (Fig. 1). For level walking, two forceplates (Advanced Mechanical Technology Inc.) located in the center of an 8-m level walkway were used to measure the GRF. Three-dimensional kinematic data of the body segments of the subjects were measured using a seven-camera motion-analysis system (Vicon512, Oxford Metrics Group, UK) at a sampling rate of $120 \mathrm{~Hz}$. All the measurements were performed synchronously.

Data collection and analysis. For the measurement of the 3D kinematic data, 39 retroreflective markers were placed on the body segments. For the pelvis-leg apparatus, 28 markers were placed over the bilateral ASIS and PSIS, greater trochanter, midthigh, medial and lateral epicondyles, head of the fibula, tibial tuberosity, medial and lateral malleoli, navicular tuberosity, second metatarsal head, fifth metatarsal base, and heel. Another 11 markers were attached on the acromial processes, lateral and medial humeral epicondyles, the ulnar styloid, the ear canal, and the C7-T1 junction. Four additional markers were placed on the transducer of the right pedal to track the motion of the transducer. To remove the inertial effects of the transducer on the measured PRF during motion, the 
instrumented pedal was unloaded and driven by the noninstrumented pedal to move at a series of pedal rates while the transducer outputs were collected and represented in terms of pedal rate. These values were then subtracted from the measured PRF during tests for subsequent dynamic analysis. Coordinates of the markers gathered during a static calibration trial were used to define the anatomical coordinate systems of each of the segments and the transducer with the positive $x$-axis directed anteriorly, the positive $y$-axis superiorly, and the positive $z$-axis to the right.

With the measured GRF, PRF, and kinematic data, inverse dynamics using Newton-Euler equations of motion were used to calculate the intersegmental internal forces and moments at the joints of the lower limbs. A cardanic rotation sequence $(Z-X-Y)$ was used to describe the rotational movements of each joint (8). Inertial properties for each body segment - namely, segment mass, center of mass, and moment of inertia-were obtained using Dempster's coefficients (29). Whole-body COM position data were calculated as the weighted sum of those of all the body segments, including head and neck, trunk, pelvis, arms, forearms, thighs, shanks, and feet. All the calculated joint moments were normalized to body weight and leg length (distance between ipsilateral ASIS and medial malleolus). The movement was assumed symmetrical, so joint angles and moments were analyzed for the right leg only. The cycle of an EE could be divided into two phases marked by two transitional points: T1, the pedal reached the most anterior position; and T2, the pedal reached the most posterior position. For comparisons with level walking, the period from $\mathrm{T} 1$ to $\mathrm{T} 2$ was defined as the stance phase, and from $\mathrm{T} 2$ to $\mathrm{T} 1$ as the swing phase.

Protocol. Each subject first performed level walking at a self-selected speed in a gait laboratory. Mean step length and cadence for each subject were then calculated from the walking trials. The parameters of the ET were then set to enable an EE similar to the measured level walking, with an average pedal rate of $50 \mathrm{rpm}$, an average stride length of $110 \%$ leg length, and no workload. The subjects were allowed to familiarize themselves with the walkway and ET before experimental data were recorded. A metronome was used to assist the subject to perform EE at the prescribed pedal rate. Data collection started when the subject reached the correct pedal rate and maintained that cadence for $5 \mathrm{~s}$. Eight cycles were then collected, and the middle three were used for subsequent dynamic analysis. GRF, PRF, COM displacements, and the angles and moments at each joint of the lower limbs during both activities were calculated, and their peak values were extracted for subsequent statistical analysis. The maximum loading rates around heelstrike of the PRF/GRF were calculated as the maximum gradient of a cubic spline fitted to the corresponding measured data. Stick figures of a typical subject with the measured PRF/ GRF vectors superimposed were generated, to demonstrate the effects of the PRF/GRF and the lower-limb posture on the joint moments for both walking and EE.
Statistical analysis. The calculated variables between the two activities were analyzed using a paired $t$-test with a significance level of 0.05. SPSS version 10.0 (SPSS Inc., Chicago, IL) was used for all statistical analyses.

\section{RESULTS}

The mean step length and cadence were $52.17 \mathrm{~cm}$ $(\mathrm{SD}=3.55)$ and 105.76 steps per minute $(\mathrm{SD}=9.53)$ during level walking, and those for EE were $50.56 \mathrm{~cm}$ $(\mathrm{SD}=2.14)$, and $52.20 \mathrm{rpm}(\mathrm{SD}=2.34)$, or 104.40 steps per minute, respectively. There was no significant difference between these two activities (step length, $P=0.130$; cadence, $P=0.616)$.

The pedal moved along an oblique ellipse with a major axis of $50.56 \pm 2.14 \mathrm{~cm}$ in length and tilted $6.19 \pm 0.36^{\circ}$ anteriorly (downward slope), and a minor axis of $17.23 \pm$ $1.19 \mathrm{~cm}$, while keeping the mediolateral center-to-center distance between the two pedals at about $25 \mathrm{~cm}$. With the movement ranges, the subjects managed to keep the motion of the COM within $1.7 \mathrm{~cm}$ anteroposteriorly, $5.1 \mathrm{~cm}$ vertically, and $6.0 \mathrm{~cm}$ mediolaterally during a complete cycle. Corresponding values for level walking were 111.0, 2.9 , and $4.7 \mathrm{~cm}$, respectively.

The patterns of the anteroposterior component of the GRF during walking and PRF during EE were similar, but those for the other two components were different (Fig. 2). The GRF was present only during the stance phase, whereas the PRF existed for the entire cycle of EE. During level walking the vertical and mediolateral reaction forces had double peaks, whereas only single peaks were observed during EE. Compared with walking, the PRF was positioned and directed more posteriorly and medially in the stance phase. In early stance, the magnitude of the vertical PRF of EE was smaller (EE: $94.44 \pm 9.00 \%$ BW, walking: $103.05 \pm 5.12 \% \mathrm{BW}, P=0.003$ ), whereas the medial (EE: $8.09 \pm 2.08 \%$ BW, walking: $4.36 \pm 2.00 \%$ BW, $P<0.0001)$ and posterior (EE: $15.83 \pm 3.13 \% \mathrm{BW}$; walking: $13.61 \pm$ $2.09 \% \mathrm{BW}, P=0.03$ ) shear forces were greater. The maximum loading rates of the vertical, mediolateral, and anteroposterior components during $\mathrm{EE}$ were $3.78 \pm 1.75$, $0.42 \pm 0.16$, and $0.85 \pm 0.50 \mathrm{BW} \cdot \mathrm{s}^{-1}$, respectively. The corresponding values for level walking were $48.78 \pm 16.98$, $4.61 \pm 2.07$, and $6.27 \pm 3.03 \mathrm{BW} \cdot \mathrm{s}^{-1}$. The maximum loading rates during $\mathrm{EE}$ were all significantly smaller than the corresponding values during walking (all components, $P<$ $0.0001)$. Stick figures of a typical subject with the measured PRF/GRF vectors superimposed are shown in Figure 3.

In the sagittal plane, the two activities showed similar angular patterns for the hip and knee, but with different amplitudes (Fig. 4). The hip and knee remained more flexed for almost the entire EE cycle. The differences for the ankle joint occurred in the second half of the movement cycle, mainly during swing (Fig. 4). Compared with walking, EE had significantly greater peak flexion angles at the hip, knee, and ankle (Table 1). In the frontal plane, the hip joint 

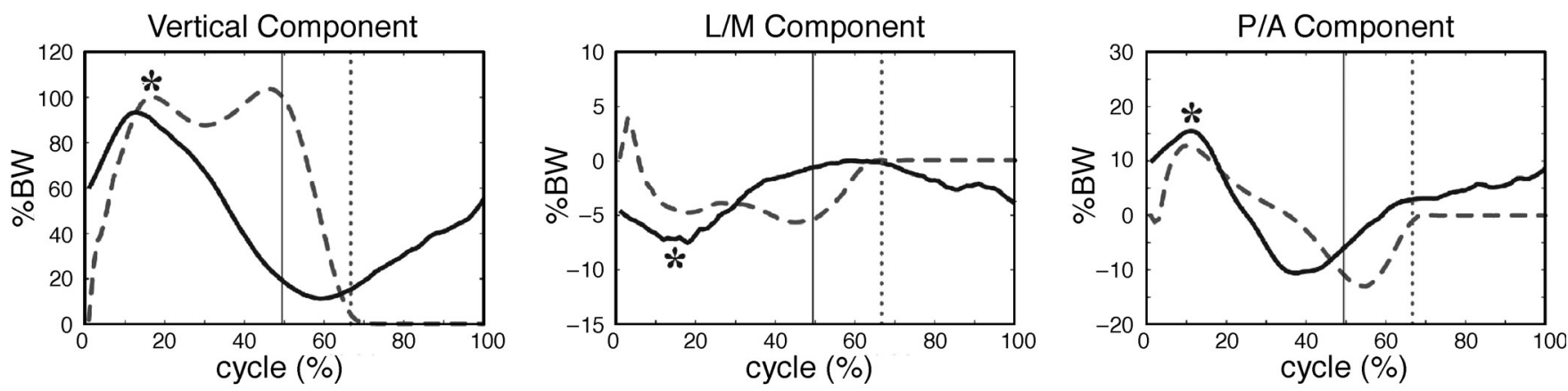

FIGURE 2-Ensemble-averaged PRF (solid lines) during elliptical exercise and GRF (dashed lines) during level walking. * Significant difference between the two activities $(P<0.05)$.

remained slightly abducted during most of the EE cycle, whereas it was adducted during the stance phase of gait (Fig. 4). The knee had similar motion patterns during the two activities, but it had significantly greater peak abduction angles during EE (Table 1). The ankle remained adducted during the late stance phase and early swing phase of EE. The angular motions of the ankle joint were different between the two activities, both in pattern and magnitude. In the transverse plane, the ankle joint remained internally rotated throughout almost the entire EE cycle, and the peak internal rotation angles were significantly greater during EE (Table 1).

In the sagittal plane, the patterns of the hip moments during EE were quite different from those during walking (Fig. 5). Flexor moments were required at the hip throughout almost the whole EE cycle. During stance, peak hip flexor moments during EE were significantly greater than those during walking, whereas peak hip extensor moments were smaller $(P=0.003)$ (Table 2). Similar patterns of the sagittal knee moments were found during the two activities, but extensor moments were needed throughout the EE cycle, and flexor moments were needed during walking (Fig. 5). Both peak knee extensor moments during EE were significantly greater than those during level walking (Table 2). The ankle required much smaller plantarflexor moments during the $\mathrm{EE}$ cycle than during level walking (Table 2).

In the frontal and transverse planes, the patterns and magnitudes of the moment components were largely different between EE and walking (Fig. 5). During stance phase, all peak abductor moments during EE were significantly smaller than those during level walking (Table 2). Peak knee internal rotation moments and ankle external rotation moments were also smaller during EE (Table 2).

\section{DISCUSSION}

The current study is arguably the first to perform a detailed 3D dynamic analysis of the lower extremities during EE and to compare the results to those during level walking. Although ET are capable of simulating the motion of human gait, differences in the lower-limb kinematics and kinetics were found between these two activities.
A

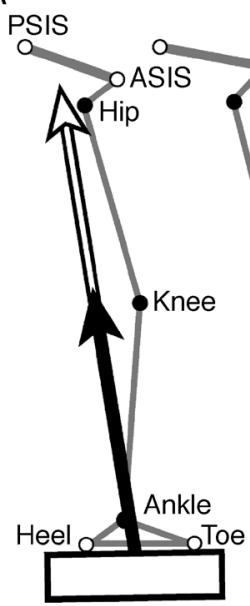

EE
B

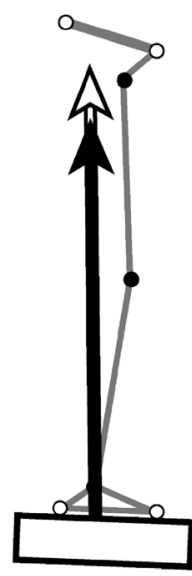

EE

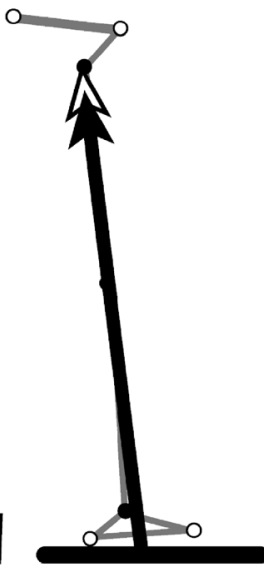

Level Walking

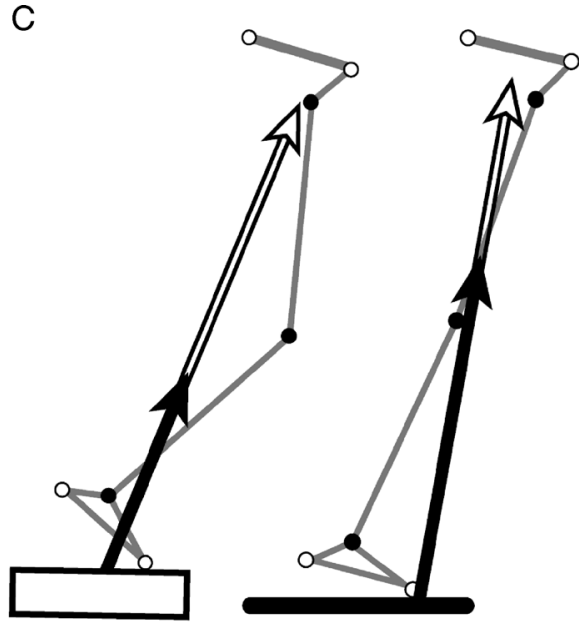

EE

Level Walking

FIGURE 3-Stick figures of the pelvis-leg apparatus of a typical subject with the PRF and GRF vectors at instances of $(A)$ heelstrike, $(B)$ peak knee extensor moment, and $(C)$ peak anterior shear force during EE and level walking. The dynamic GRF and PRF vectors are shown as filled arrows, and open arrows with magnitudes equal to the body weight are superimposed along each GRF/PRF vector for reference. Joint centers are shown as filled circles, and bony landmarks for drawing the stick figures are shown as open circles. 
A Hip Flex/Ext
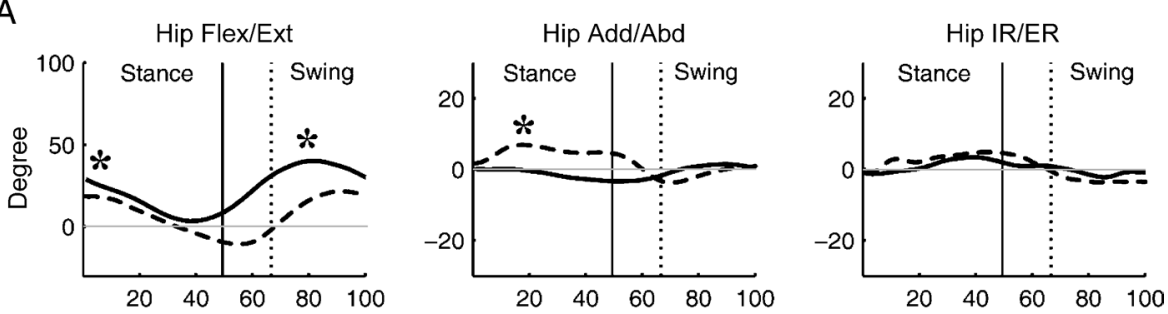

B
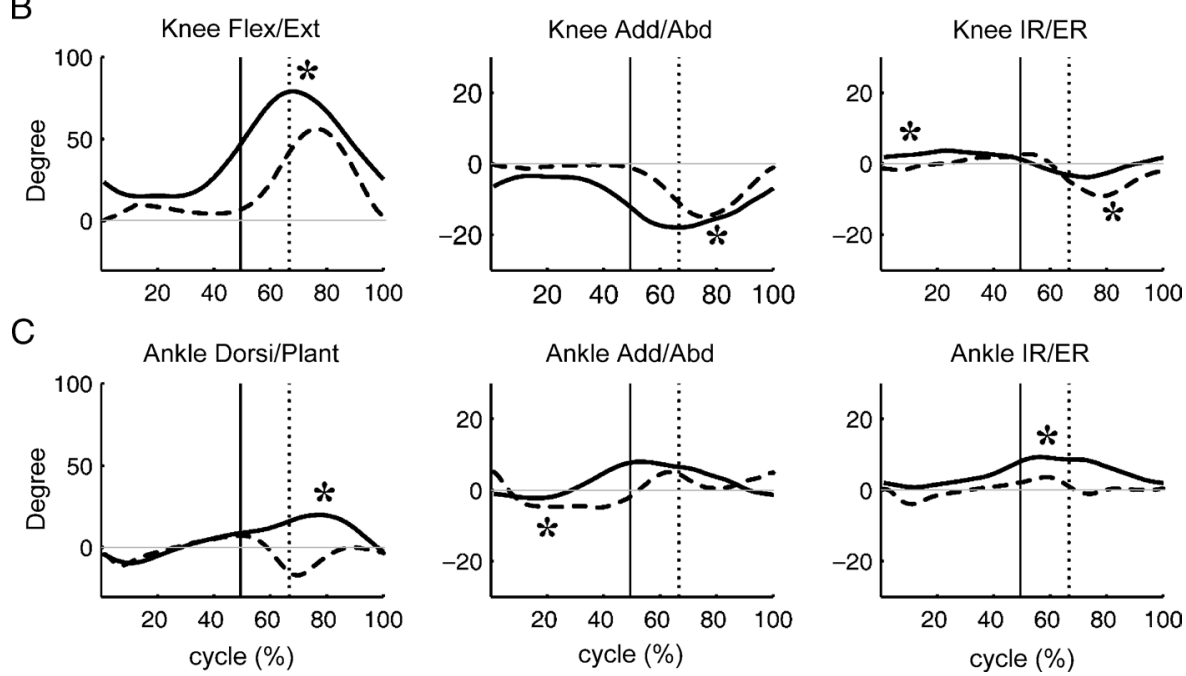

FIGURE 4-Ensemble-averaged three-dimensional joint angles at the $(A)$ hip, $(B)$ knee, and $(C)$ ankle joints during elliptical exercise (solid lines) and level walking (dashed lines). * Significant difference between the two activities $(P<0.05)$.

The closed kinetic chain motion of the locomotor system with the constrained pedal trajectory during EE seemed to be the main reason for the observed differences in the kinematics and kinetics compared to level walking. During level walking, the swing limb is free of constraints for limb advancement, whereas changes of the joint kinematics of the lower limbs were needed during EE to ensure that the swing foot followed the trajectory of the swing pedal. Even with the considerable range of motion of all the lower-limb joints, all the subjects successfully reached a consistent kinematic pattern of the joints that kept the COM excursion within a region that was much smaller than allowed. The

TABLE 1. Means and standard deviations (SD) of the peak angles during level walking and elliptical exercise $\left({ }^{*} P<0.05\right)$.

\begin{tabular}{|c|c|c|c|c|c|}
\hline \multirow[b]{2}{*}{ Angle $\left({ }^{\circ}\right)$} & \multicolumn{2}{|c|}{ EE } & \multicolumn{2}{|c|}{ Walking } & \multirow[b]{2}{*}{$P$} \\
\hline & Mean & SD & Mean & SD & \\
\hline \multicolumn{6}{|l|}{ Hip } \\
\hline \multicolumn{6}{|l|}{ Stance } \\
\hline Flexion & 28.89 & 7.41 & 19.43 & 3.79 & $<0.0001^{*}$ \\
\hline Adduction & 0.95 & 2.66 & 7.38 & 1.95 & $<0.0001^{*}$ \\
\hline Internal rotation & 4.19 & 3.96 & 5.74 & 4.44 & 0.087 \\
\hline \multicolumn{6}{|l|}{ Swing } \\
\hline Flexion & 40.33 & 7.33 & 21.91 & 3.96 & $<0.0001^{*}$ \\
\hline Abduction & 4.32 & 3.58 & 4.45 & 2.22 & 0.849 \\
\hline External rotation & 3.07 & 5.27 & 5.56 & 4.85 & 0.070 \\
\hline \multicolumn{6}{|l|}{ Knee } \\
\hline \multicolumn{6}{|l|}{ Stance } \\
\hline Internal rotation & 4.51 & 2.37 & 3.32 & 2.49 & 0.094 \\
\hline \multicolumn{6}{|l|}{ Swing } \\
\hline Flexion & 79.43 & 7.43 & 57.49 & 6.27 & $<0.0001^{*}$ \\
\hline Abduction & 18.38 & 6.08 & 15.44 & 5.38 & $0.001^{*}$ \\
\hline External rotation & 4.32 & 4.84 & 10.26 & 3.66 & $<0.0001^{\star}$ \\
\hline \multicolumn{6}{|l|}{ Ankle } \\
\hline \multicolumn{6}{|l|}{ Total cycle } \\
\hline Dorsiflexion & 21.15 & 7.15 & 7.56 & 2.67 & $<0.0001^{\star}$ \\
\hline Internal rotation & 9.79 & 3.98 & 4.72 & 1.78 & $<0.0001^{*}$ \\
\hline \multicolumn{6}{|l|}{ Stance } \\
\hline Plantarflexion & 10.00 & 5.44 & 11.26 & 2.71 & 0.339 \\
\hline Abduction & 2.63 & 3.93 & 5.65 & 2.33 & $0.001^{*}$ \\
\hline
\end{tabular}


Hip Ext/Flex

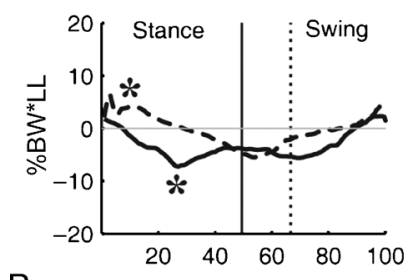

B

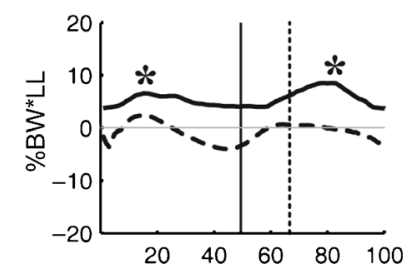

C

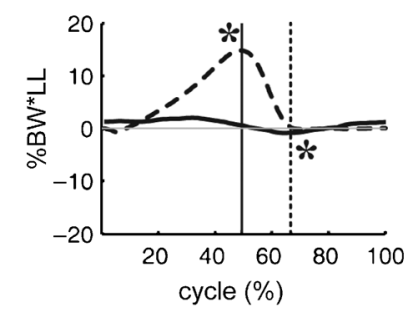

Hip Abd/Add

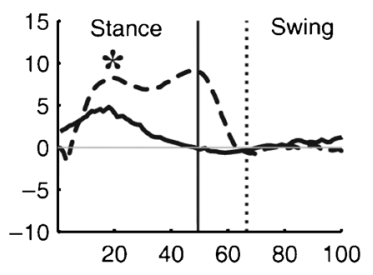

Knee Abd/Add

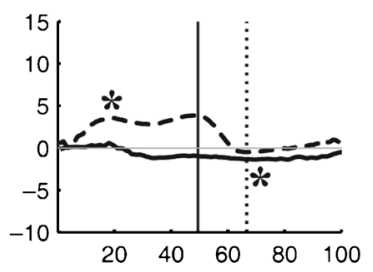

Ankle Abd/Add

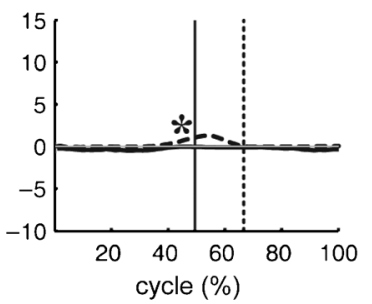

Hip IR/ER

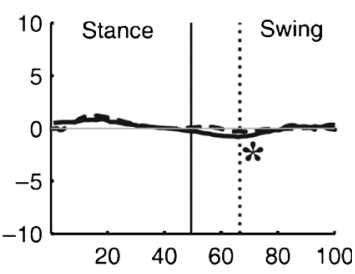

Knee IR/ER

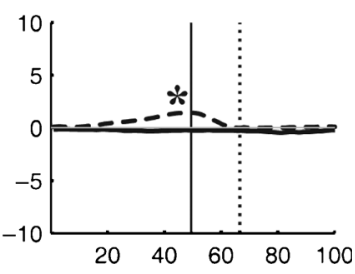

Ankle IR/ER

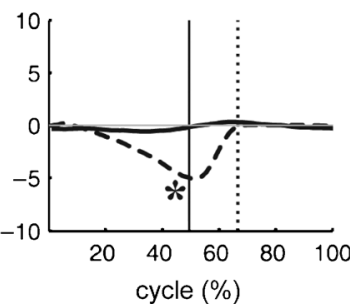

FIGURE 5-Ensemble-averaged three-dimensional joint moments at the $(A)$ hip, $(B)$ knee, and $(C)$ ankle during elliptical exercise (solid lines) and level walking (dashed lines). * Significant difference between the two activities $(P<0.05)$.

25-cm mediolateral center-to-center distance between the two pedals suggests that the COM could shift between the two feet by the same amount. However, the continuously abducted hip and knee and the adducted and internally rotated ankle of the stance limb helped to reduce the COM's mediolateral displacements to an average of $6.0 \mathrm{~cm}$ (Fig. 4). Even though the ET has a vertical pedal displacement of $17 \mathrm{~cm}$ and a step length of

TABLE 2. Means and standard deviations (SD) of the peak moments during level walking and elliptical exercise $\left({ }^{*} P<0.05\right)$.

\begin{tabular}{|c|c|c|c|c|c|}
\hline \multirow{2}{*}{$\begin{array}{l}\text { Moments } \\
\text { (!\% BW·LL) }\end{array}$} & \multicolumn{2}{|c|}{$\mathrm{EE}$} & \multicolumn{2}{|c|}{ Walking } & \multirow[b]{2}{*}{$P$} \\
\hline & Mean & SD & Mean & SD & \\
\hline \multicolumn{6}{|l|}{ Hip } \\
\hline \multicolumn{6}{|l|}{ Stance } \\
\hline Flexor & 8.55 & 3.42 & 6.19 & 1.45 & $0.012^{\star}$ \\
\hline Extensor & 2.05 & 3.80 & 7.08 & 3.05 & $0.003^{\star}$ \\
\hline Abductor & 5.17 & 2.49 & 8.47 & 1.11 & $0.0001^{*}$ \\
\hline Internal rotator & 1.01 & 0.50 & 1.28 & 0.51 & 0.127 \\
\hline \multicolumn{6}{|l|}{ Swing } \\
\hline Flexor & 6.56 & 1.73 & 4.15 & 6.49 & 0.198 \\
\hline Adductor & 1.05 & 0.82 & 2.25 & 2.32 & 0.078 \\
\hline External rotator & 1.08 & 0.67 & 0.61 & 0.31 & $0.037^{\star}$ \\
\hline \multicolumn{6}{|l|}{ Knee } \\
\hline \multicolumn{6}{|l|}{ Stance } \\
\hline Extensor & 7.51 & 3.08 & 2.55 & 3.04 & $0.001^{\star}$ \\
\hline Abductor & 1.31 & 1.56 & 3.69 & 0.85 & $0.0002^{\star}$ \\
\hline Internal rotator & 0.06 & 0.27 & 1.50 & 0.63 & $<0.0001^{*}$ \\
\hline \multicolumn{6}{|l|}{ Swing } \\
\hline Extensor & 9.54 & 2.00 & 1.06 & 0.31 & $<0.0001^{*}$ \\
\hline Adductor & 2.20 & 0.97 & 0.95 & 0.82 & $0.003^{\star}$ \\
\hline \multicolumn{6}{|l|}{ Ankle } \\
\hline \multicolumn{6}{|l|}{ Stance } \\
\hline Plantarflexor & 2.20 & 0.72 & 15.32 & 1.62 & $<0.0001^{*}$ \\
\hline Abductor & 0.21 & 0.35 & 1.43 & 0.67 & $<0.0001^{*}$ \\
\hline External rotator & 0.69 & 0.22 & 5.40 & 1.08 & $<0.0001^{*}$ \\
\hline \multicolumn{6}{|l|}{ Swing } \\
\hline Dorsiflexor & 1.04 & 0.53 & 0.20 & 0.07 & $<0.0001^{*}$ \\
\hline Adductor & 0.63 & 0.34 & 0.09 & 0.15 & $0.0002^{*}$ \\
\hline
\end{tabular}


$50 \mathrm{~cm}$, the vertical and anteroposterior displacements of the COM were kept at about 5.1 and $1.7 \mathrm{~cm}$, respectively. This was achieved by a greater flexion of the hip and knee, as well as by greater dorsiflexion at the ankle during the EE cycle. This more flexed posture of both the lower limbs seemed to be useful for the reduction of the COM displacements. The swing limb also contributed in reducing the vertical PRF by assisting in supporting the body weight during the exercise (Fig. 2). This is also related to the reduction of the loading rate during EE. Although smaller vertical PRF helped reduce joint moments in the frontal and transverse planes, the specific kinematic changes increased the sagittal components of the lever arms available to the PRF with the lines of action of the PRF passing away from the joint centers (Fig. 3), increasing the sagittal components of the joint moments (Table 2).

Although a small loading rate at heelstrike during EE may reduce the risk of developing tibiofemoral joint osteoarthritis, the knee joint sustained a continuously greater extensor moment at a greater flexion angle during EE, with the peak moments approximately three times greater than those during walking. A greater knee extensor moment indicates increased loading for the quadriceps muscles (19) and this loading would be further increased with increased knee flexion as the effective lever arm lengths decreased with increasing knee flexion (13). This increase may lead to early fatigue of the muscles, which could in turn limit the duration, and consequently the effects, of fitness training. Greater loading of the quadriceps at greater knee flexion during EE may be important with respect to the disadvantageous loads within the patellofemoral compartment. Previous theoretical and experimental studies have shown that patellofemoral joint contact force increased with increased quadriceps force and knee flexion angle $(1,30)$. It seems that the use of ET for athletic and rehabilitative training would have to consider users' knee joint function and muscle strength to avoid any unnecessary injuries.

Reducing the slope of the pedal ellipse may be helpful for the reduction of the knee joint extensor moment. In the present study the pedal moved along an oblique ellipse with the major axis tilted anteriorly. This downslope inclination may be associated with the observed greater posterior shear force and greater knee flexion (Figs. 2-4). The increased posterior shear force shifted the line of action of the PRF more posterior to the hip and knee joint centers with increased lever-arm lengths (Fig. 3), contributing to the greater hip flexor and knee extensor moments (Table 2). Similar phenomena were also reported in previous comparative studies on level and slope walking $(17,26)$. Lay et al. (17) showed that flexion angles and extensor moments at the knee increased with increasing downward slopes. Because Lay et al. (17) studied slopes steeper than $15^{\circ}$, whereas the slope of the pedal ellipse in the present study was about $6^{\circ}$, further study is needed for determining to what extent the reduction of the slope of the pedal ellipse can affect the joint angles and moments.
Improvement of the mobility of the pedal system of the ET may present a possibility of reducing hip and knee moments. Apart from the greater magnitudes, the observed motion patterns of the hip and knee during EE were similar to those during walking. However, the ankle had very different motion patterns, especially during late stance and swing phase (Fig. 4). During walking, the heel, ankle, and forefoot serve as rockers to reduce the vertical excursion of the COM while in progression, resulting in alternate arcs of dorsiflexion and plantarflexion at the ankle (27). During the late stance of EE, although the forefoot rocker existed, the excessive flexion at the hip and knee caused the tibia to rotate more than the foot such that the ankle joint continued to dorsiflex instead of plantarflex as in level walking (Fig. 4). These kinematic differences change the positions of the joint centers, lines of action of the PRF, and, thus, the moments at the lower-limb joints (Fig. 3). A reduced ankle plantarflexor moment, less than $15 \%$ of the peak moment during walking (Table 2), was accompanied by increased moments at the hip and knee during EE. If the ankle motion could be brought back to patterns similar to level walking, such as through the change of the mobility of the pedal system, the differences in lower-limb-joint moments between EE and walking may be reduced. Pierson-Carey et al. (18) have shown that mobility of the ankle affects the magnitude and orientation of the resultant PRF during pedaling. Previous studies on cycling also suggest that increasing the mobility of the pedal system helps improve the efficiency of the knee and hip power transfer $(15,18)$, alleviating the loads transmitted through the knee joint, thus reducing overuse knee injuries $(5,20)$. Ruby and Hull (20) have revealed that permitting relative motion of the pedal would eliminate constraint loads at the pedal and, thus, decrease intersegmental loads at the knee joint, whereas Boyd et al. (5) report that the valgus knee moment was significantly reduced by a dual-rotation platform (both adduction/abduction and inversion/eversion) compared with the fixed or inversion/eversion-only design. Increasing the mobility of the pedal system increases the degrees of freedom of the closed kinetic chain of the EE and reduces the constraints to the feet. Because of the similarities between EE and standing cycling, it seems that appropriate mobility of the pedal system of the ET may be helpful for a reduction of the potentially harmful loading at the knee.

\section{CONCLUSIONS}

The current study showed that smaller vertical PRF and loading rates during simulated walking on an ET were achieved at the expense of greater hip flexor and knee extensor moments. The joint kinematics and dynamics of the lower limbs during EE appeared to result from the closed kinetic chain motion of the locomotor system with the constrained pedal trajectory. Use of the ET for athletic 
and rehabilitative training would have to consider users' joint function and muscle strength, especially at the knee, to avoid injuries. Future designs of ET may consider improving the mobility of the pedal system and reducing the slope of the pedal ellipse to reduce harmful joint loadings.

\section{REFERENCES}

1. Ahmed, A. M., and D. L. Burke. In-vitro measurement of static pressure distribution in synovial joints - part I: tibial surface of the knee. J. Biomech. Eng. 105:216-225, 1983.

2. American College of Sports Medicine. ACSM's Guidelines for Exercise Testing and Prescription. Philadelphia, PA: Lippincott Williams \& Wilkins, 2000.

3. Batte, A. L., J. Darling, J. Evans, L. M. Lance, E. I. Olson, and D. M. Pincivero. Physiologic response to a prescribed rating of perceived exertion on an elliptical fitness cross-trainer. J. Sports Med. Phys. Fitness 43:300-305, 2003.

4. Beckett, R., and K. Chang. An evaluation of the kinematics of gait by minimum energy. J. Biomech. 1:147-159, 1968

5. Boyd, T. F., R. R. Neptune, and M. L. Hull. Pedal and knee loads using a multi-degree-of-freedom pedal platform in cycling. J. Biomech. 30:505-511, 1997.

6. Callaghan, M. J. Lower body problems and injury in cycling. J. Bodywork Mov. Ther. 9:226-236, 2005.

7. Chang, Y. H., H. W. C. Huang, C. M. Hamerski, and R. Kram. The independent effects of gravity and inertia on running mechanics. J. Exp. Biol. 203:229-238, 2000.

8. Cole, G. K., B. M. Nigg, J. L. Ronsky, and M. R. Yeadon. Application of the joint coordinate system to three-dimensional joint attitude and movement representation: a standardization proposal. J. Biomech. Eng. 115:344-349, 1993.

9. Collins, J. J., and M. W. Whittle. Impulsive forces during walking and their clinical implications. Clin. Biomech. (Bristol, Avon) 4:179-187, 1989.

10. Cuff, D. J., A. Iganszewski, G. S. Meneilly, H. D. Tildesley, A. Martin, and J. J. Frohlich. Effective exercise modality to reduce insulin resistance in women with type 2 diabetes. Diabetes Care 26:2977-2982, 2003.

11. Egana, M., and B. Donne. Physiological changes following a 12 week gym based stair-climbing, elliptical trainer and treadmill running program in females. J. Sports Med. Phys. Fitness 44: 141-146, 2004.

12. Ganley, T. J., R. L. Gaugles, and L. A. Moroz. Consultation with the specialist: patellofemoral conditions in childhood. Pediatr. Rev. 27:264-269, 2006.

13. Gill, H. S., and J. J. O'ConNor. Biarticulating two-dimensional computer model of the human patellofemoral joint. Clin. Biomech. (Bristol, Avon) 11:81-89, 1996.

14. Green, J. M., T. R. Crews, R. C. Pritchett, C. Mathfield, and L. HALl. Heart rate and ratings of perceived exertion during treadmill and elliptical exercise training. Percept. Mot. Skills 98:340-348, 2004.

15. Gregor, S. M., K. L. Perell, S. Rushatakankovit, E. Miyamoto, R. Muffoletto, and R. J. Gregor. Lower extremity general
The authors would like to thank the Cycling \& Health Tech Industry R\&D Center for supporting the work reported in this paper.

The authors declare no proprietary, financial, professional, or other personal interest of any nature or kind in any product, service, and/or company that could be construed as influencing the position presented in this article.

muscle moment patterns in healthy individuals during recumbent cycling. Clin. Biomech. (Bristol, Avon) 17:123-129, 2002.

16. Kravitz, L., B. Wax, J. J. Mayo, R. Daniels, and K. Charette. Metabolic response of elliptical exercise training. Med. Sci. Sports Exerc. 30:S169, 1998.

17. Lay, A. N., C. J. Hass, and R. J. Gregor. The effecs of sloped surfaces on locomotion: a kinematic and kinetic analysis. $J$ Biomech. 39:1621-1628, 2006.

18. Pierson-Carey, C. D., D. A. Brown, and C. A. Dairaghi. Changes in resultant pedal reaction due to ankle immobilization during pedaling. J. Appl. Biomech. 13:334-346, 1997.

19. Reilly, D. T., and M. Martens. Experimental analysis of the quadriceps muscle force and patello-femoral joint reaction force for various activities. Acta Orthop. Scand. 43:126-127, 1972.

20. Ruby, P., and M. L. Hull. Response of intersegmental knee loads to foot/pedal platform degrees of freedom in cycling. J. Biomech. 26:1327-1340, 1993

21. Shelbourne, K. D., T. D. Henne, and T. Gray. Recalcitrant patellar tendinosis in elite athletes: surgical treatment in conjunction with aggressive postoperative rehabilitation. Am. J. Sports Med. 34:1141-1146, 2006.

22. Simons, R., and R. Andel. The effects of resistance training and walking on functional fitness in advanced old age. J. Aging Health 18:91-105, 2006

23. Sporting Goods Manufacturers Association. Sports Participation Topline Report. Washington, DC: Sporting Goods Manufacturers Association, 2006.

24. Stalzer, S., M. Wahoff, M. Scanlan, and P. Dravovitch. Rehabilitation after hip arthroscopy. Oper. Tech. Orthop. 15: 280-289, 2005.

25. Steadman, J. R., and T. S. Bollom. Principles of ACL revision surgery and rehabilitation. Sports Med. Arthrosc. 13:53-58, 2005.

26. Sun, J., M. Walters, N. Svensson, and D. Lloyd. The influence of surface slope on human gait characteristics: a study of urban pedestrains walking on an inclined surface. Ergonomics 39: 677-692, 1996.

27. Sutherland, D. H., R. Olshen, L. Cooper, and S. L.-Y. Woo. The development of mature gait. J. Bone Joint Surg. Am. 62A: 336-353, 1980

28. Voloshin, A. The influence of walking speed on dynamic loading on the human musculoskeletal system. Med. Sci. Sports Exerc. $32: 1156-1159,2000$

29. Winter, D. A. Biomechanics and Motor Control of Human Movement. New York, NY: John Wiley \& Sons, 2005.

30. Yamaguchi, G. T., and F. E. Zajac. A planar model of the knee joint to characterize the knee extensor mechanism. J. Biomech. 22:1-10, 1989. 